\title{
DISEÑO DE UNA MÁQUINA PARA CORTAR Y DOBLAR ESTRIBOS EN SERIE PARA COLUMNAS DE HORMIGÓN ARMADO
}

\author{
DESIGN A MACHINE TO CUT AND BEND \\ BRACKETS IN A SERIAL ORDER FOR \\ REINFORCED CONCRETE PILLARS
}

\author{
José Olger Pérez-Silva ${ }^{1, *}$, Oscar Paul Trujillo-Zurita ${ }^{2}$
}

\section{Resumen}

En este artículo se presenta el diseño y construcción de una máquina para cortar y doblar estribos para columnas de hormigón armado. Esta es una solución que busca reemplazar el trabajo manual que realiza una persona para la fabricación de estos elementos. Parte desde el análisis del índice RULA, (Rapid Upper Limb Assessment) que determina la carga de afectación ocupacional a la salud del operario, quien de forma artesanal produce 600 estribos diarios. El resultado obtenido fue de 7 y ante esta situación se plantea una alternativa de trabajo al diseñar y posteriormente construir un equipo que realice las mismas operaciones reemplazando el esfuerzo humano. Se describe cómo fue el proceso de diseño y fabricación de la máquina que permitió obtener los mismos 600 estribos en 2 horas de funcionamiento y sin causar un alto grado de agotamiento físico en la persona, situación que se hace notoria cuando el índice RULA baja de 7 a 3 .

Palabras clave: enfermedad ocupacional, estribo, estructura, mecanismo, productividad. RULA (Evaluación Rápida de los Miembros Superiores).

\section{Abstract}

This article presents the design and construction of a machine that is used to cut and bend braces for reinforced concrete columns. This is a solution to replace the manual work carried out by a person during the manufacturing of these elements. This project is based on analyses of R.U.L.A (Rapid Upper Limb Assessment) that determines the occupational affections in the worker's health who produces about 600 braces daily in a traditional way. The result obtained was 7. This proposal shows an alternative to design and build equipment that will realize the same operations, so that the human effort will be reduced. This article describes the process of the design and construction of the machine, the result shows the benefit of the machine that will avoid the physical effort of the worker, which is evident applying the R.U.L.A that is from $7-3$ when we obtained 600 braces in only two hours.

Keywords: Occupational safety and health, reinforcement bar, Structure, Mechanism, Productivity, R.U.L.A (Rapid Upper Limb Assessment).

\footnotetext{
1,* Carrera de Ingeniería Mecánica, Universidad Politécnica Salesiana, sede Quito - Ecuador. Autor para correspondencia : jperez@ups.edu.ec

${ }^{2}$ Departamento de Proyectos y Mejoras Industriales, Zaimella del Ecuador.
}

Recibido: 02-02-2016, aprobado tras revisión: 19-09-2016

Forma sugerida de citación: Pérez-Silva, J.; Trujillo-Zurita, O. (2016). «Diseño de una máquina para cortar y doblar estribos en serie para columnas de hormigón armado». InGENIUS. N. ${ }^{\circ} 16$, (Julio-Diciembre). pp. 5-11. ISSN: 1390-650X. 


\section{Introducción}

El crecimiento de la construcción civil donde se utilizan columnas de hormigón armado aumenta cada año (Figura 1) según el índice general de la construcción publicado por el INEC (Instituto Nacional de Estadística y Censos).

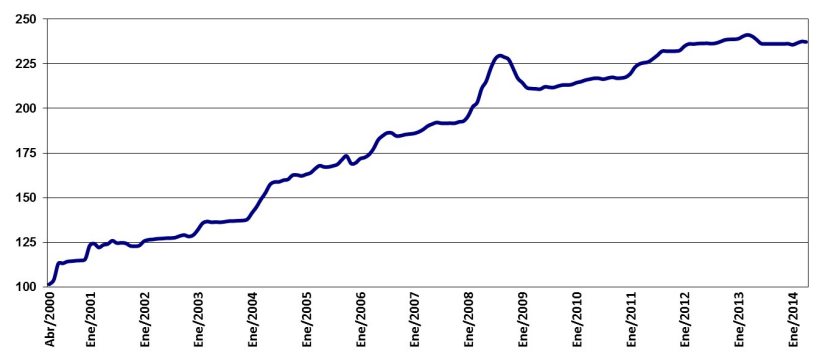

Figura 1. Censo del crecimiento anual de la construcción civil en general [1]

El principio de estabilidad y firmeza de las columnas se basa en la unión de los estribos con las varillas longitudinales, los mismos que se colocan en una cantidad aproximada de veinte por cada 2,5 metros de columna, que es la altura más común entrepisos y la medida más vendida en el mercado.

Las columnas de hormigón armado son elementos que se forman a partir de la unión de varillas largas con estribos o refuerzos (según norma internacional ACI318) que se cruzan transversalmente (Figura 2), estos elementos generalmente se los realiza en obra, lo que incrementa el tiempo de trabajo del obrero y disminuye tiempo en la construcción, situación que afecta directamente a los proyectos finales, además, causa cansancio y agotamiento en quién realiza esta operación.

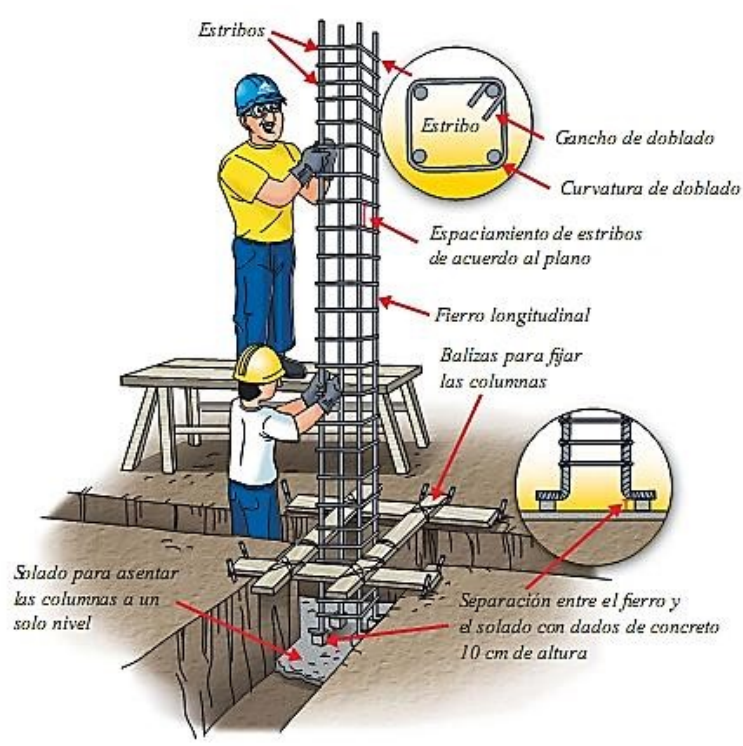

Figura 2. Armazón de hierro para columna de hormigón armado [2]
Generalmente, estos estribos los realiza un obrero en forma manual (Figura 3) con una producción aproximada de 600 unidades en una jornada laboral de 8 horas, lo que implica materia prima para 30 columnas diarias. Al ser este un proceso repetitivo y continuo puede provocar enfermedades ocupacionales al trabajador como tenosinovitis, bursitis, trastornos musculares, etc.

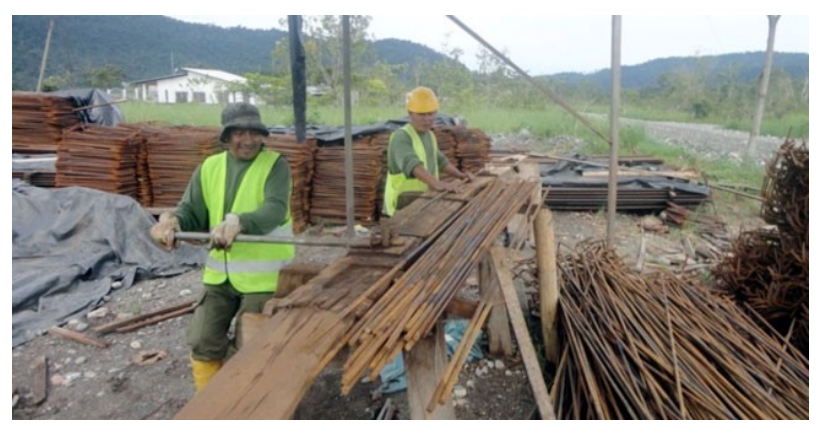

Figura 3. Producción manual [3]

Según análisis realizado aplicando el método RULA (Rapid Upper Limb Assessment, que en español quiere decir Evaluación Rápida de los Miembros Superiores) se ha determinado que este procedimiento de trabajo da una puntuación final de 7 (Figura 4) que interpretado recomienda estudiar y modificar inmediatamente el proceso operativo.

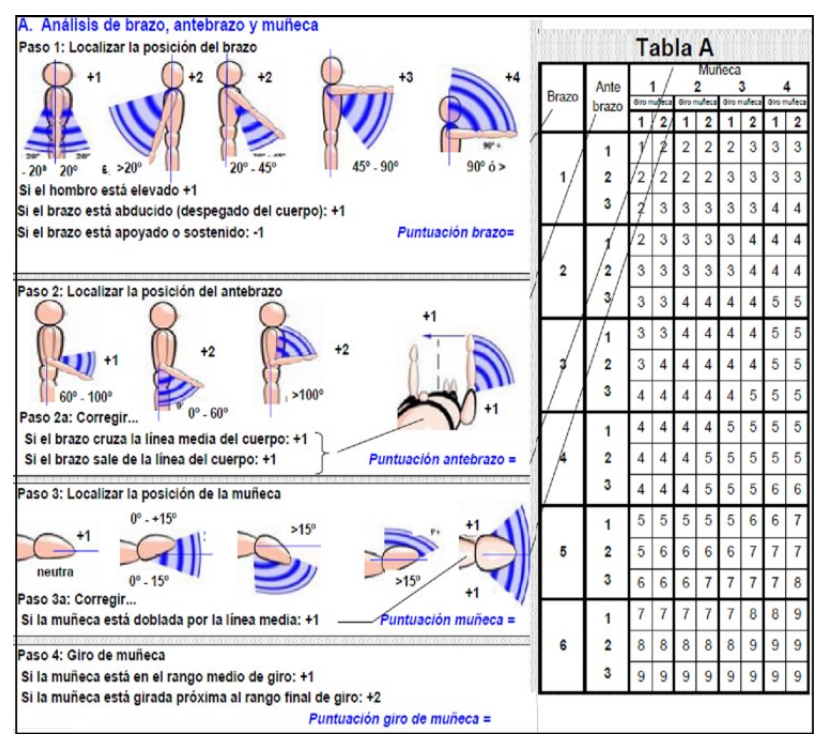

Figura 4. Hoja de análisis Método RULA [4]

Analizando esos resultados que afectan directamente a la salud del operario y en busca de la solución a esas condiciones extremas de trabajo, se propuso investigar una forma alternativa de realizar dicha labor y se obtuvo como resultado, el diseño y la posterior construcción de una máquina (Figura 5) para cortar y doblar estribos en serie, con lo que se disminuiría el mencionado índice y que, además, se incrementaría 
la producción diaria cual lo cual se traduciría tanto en beneficio social como económico al disminuir enfermedades profesionales y costos de producción respectivamente.

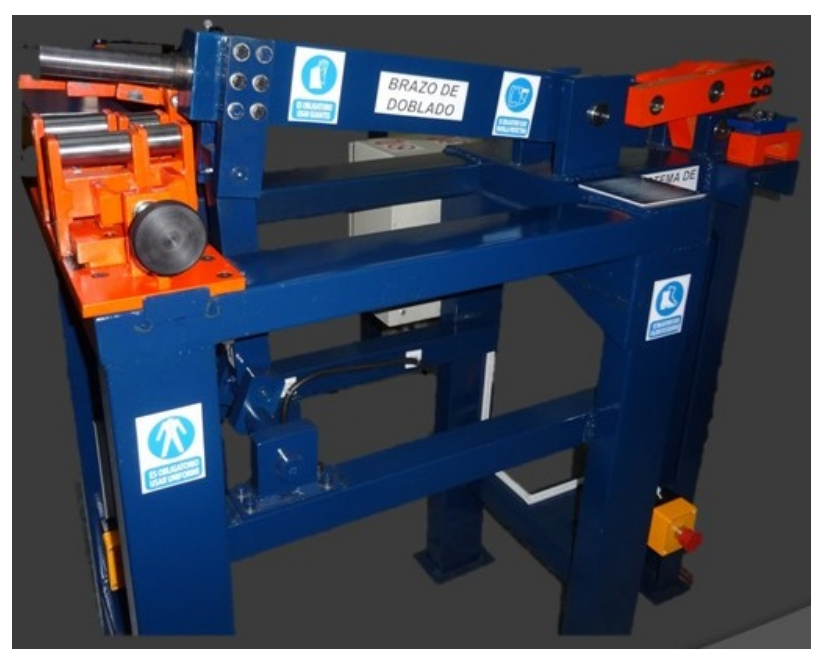

Figura 5. Máquina para cortar y doblar estribos

\section{Desarrollo}

\subsection{Varilla corrugada}

Una varilla corrugada es una aleación de acero y otros componentes químicos que le dan a esta características especiales, formada por corrugas o resaltos que son necesarios para la adhesión del hormigón, no tienen una capa protectora ante la corrosión u oxidación ya que el mismo concreto se encarga de revestirla y darle un tiempo de vida extendido.

Esta tiene características especiales, muy buena ductilidad, gran límite de fluencia y un alto grado de resistencia sísmica. Datos reales de ensayos realizados por la fábrica ANDEC en sus laboratorios (Tabla 1) han sido utilizados para el desarrollo del proyecto.

Tabla 1. Propiedades mecánicas de la varilla corrugada

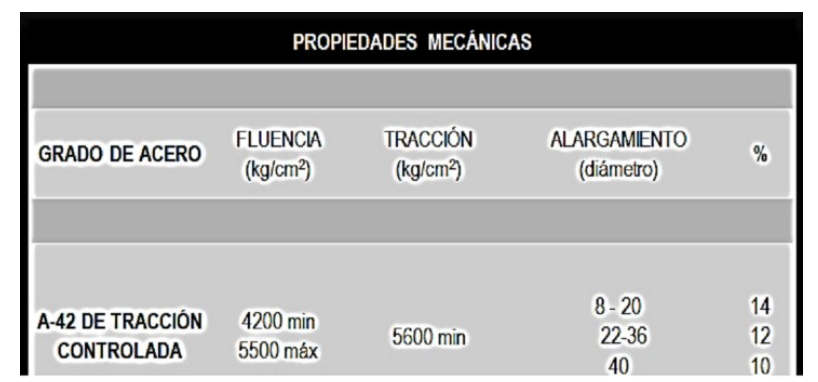

\subsection{Diseño y construcción}

El diseño de la máquina inicia con el cálculo de la fuerza necesaria para doblar la varilla corrugada (1), para lo cual se utilizó la fórmula de flexión (2) y se la combinó con el factor plástico (3), ya que se desea deformar totalmente el elemento.

$$
\begin{gathered}
F=\frac{4 \cdot K \cdot \sigma_{m a ́ x} \cdot I}{L \cdot c} \\
\sigma_{m a ́ x}=\frac{M \cdot c}{I} \\
K=\frac{M_{p}}{M}
\end{gathered}
$$

Donde las principales variables son el esfuerzo máximo de fluencia, la inercia, longitud de apoyos, distancia del eje neutro y el factor $\mathrm{K}$, el cual está definido como el factor plástico de una sección transversal. Con esta fórmula se determina inicialmente la fuerza de doblado, pero no se consigue el resultado esperado. Realizando un experimento que consiste en la aplicación de pesos a un mecanismo de palanca, se determina el factor de doblado $(\mathrm{fd}=1,32)$, definiendo así la fuerza total.

El corte de la varilla se deduce realizando una analogía del cálculo de pernos en cortante directo (4), interpretando que el esfuerzo no será el de fluencia, sino el de tracción ya que se requiere la falla del material. Para lo cual se comprueba con la fórmula de corte de chapa (5) cambiando la longitud y sección de corte por el diámetro de la varilla.

$$
\begin{gathered}
\tau=\frac{V}{A} \\
F=\sigma_{c} \cdot e \cdot l
\end{gathered}
$$

Realizado el cálculo de corte y doblado se procede con el diseño del mecanismo (Figura 6) y el cálculo de fuerzas y reacciones que se denotan en el cuerpo libre que este describe (Figura 7), determinando así las fuerzas más importantes que serían la motriz y la de corte, concluyendo que la fuerza de corte satisface la necesidad y la motriz es proporcional al torque que provee el motor reductor.

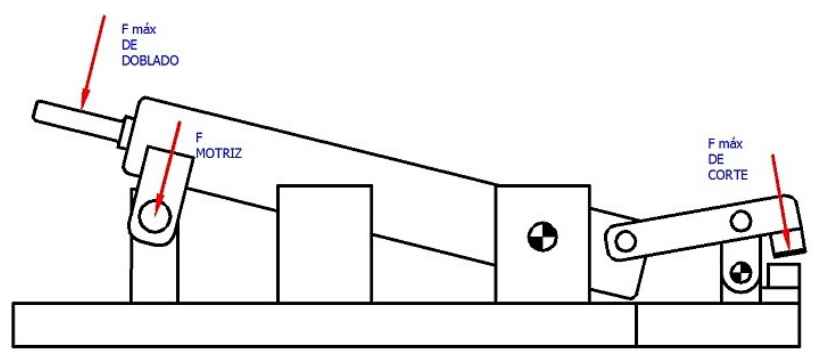

Figura 6. Mecanismo de trabajo y fuerzas significativas 


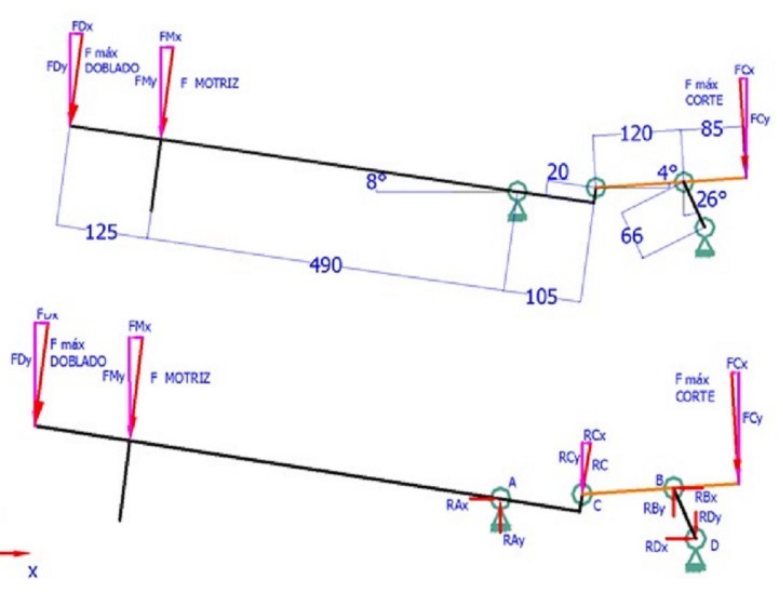

Figura 7. Diagramas de cuerpo libre.

Una de las principales ecuaciones que se utilizó para calcular el $85 \%$ de los elementos y partes es la fórmula de la flexión [5] (Figura 8), con la cual se determinó los esfuerzos de los materiales a partir de las diferentes secciones transversales utilizando tablas de Excel con la finalidad de facilitar el trabajo de iteraciones.

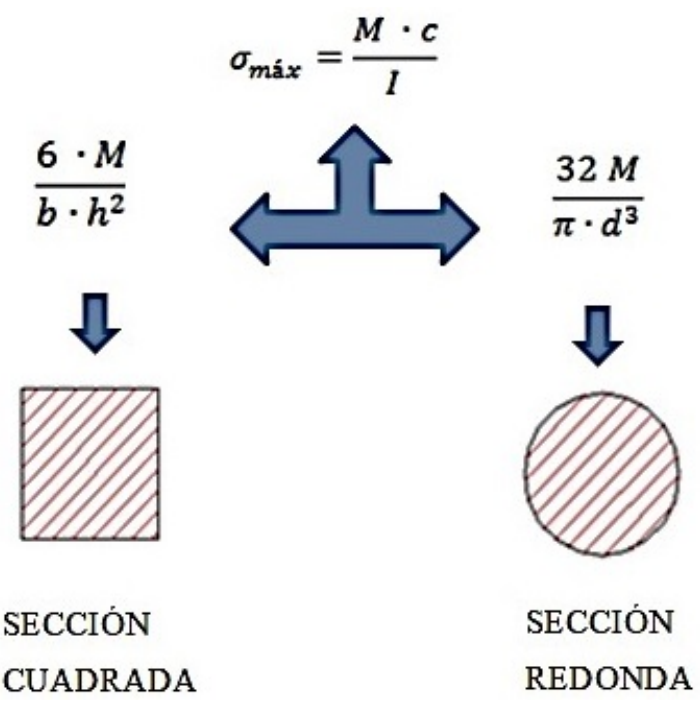

Figura 8. Fórmula de la flexión desglosada

Elementos de sección rectangular o cuadrada como eslabones, se determinaron mediante un cálculo de esfuerzos combinados (Figura 9), utilizando las fórmulas denotadas en la Tabla 2 que define el factor de concentración de esfuerzos que se crean en una sección transversal con variaciones en su superficie.

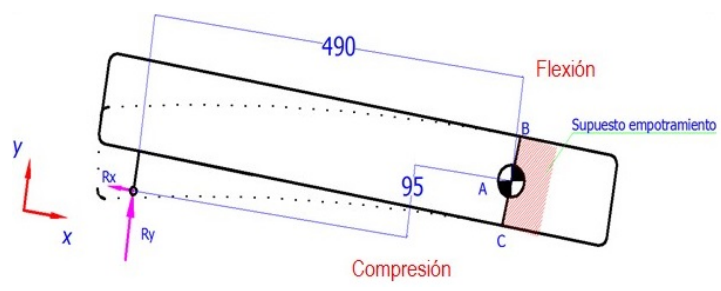

Figura 9. Fuerzas aplicadas en brazo de doblado
Tabla 2. Fórmulas de esfuerzos combinados y factor de concentración de esfuerzos $[6]$
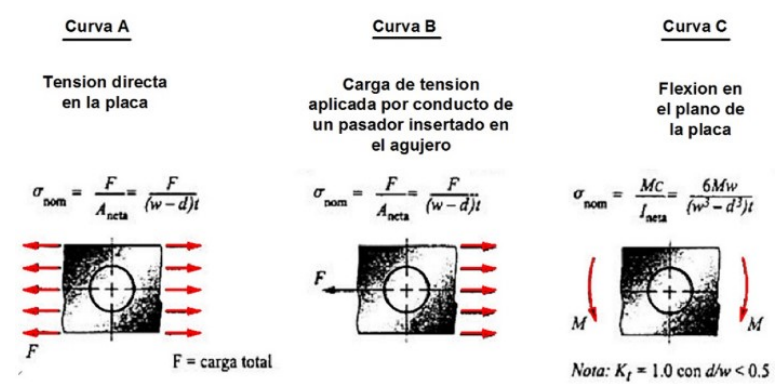

Combinando estas ecuaciones de flexión y tracción se realiza un cálculo de esfuerzo por iteraciones ejecutadas en una tabla (Tabla 3) de Excel definiendo la sección adecuada para el trabajo requerido.

Tabla 3. Cálculo de esfuerzos combinados por iteraciones

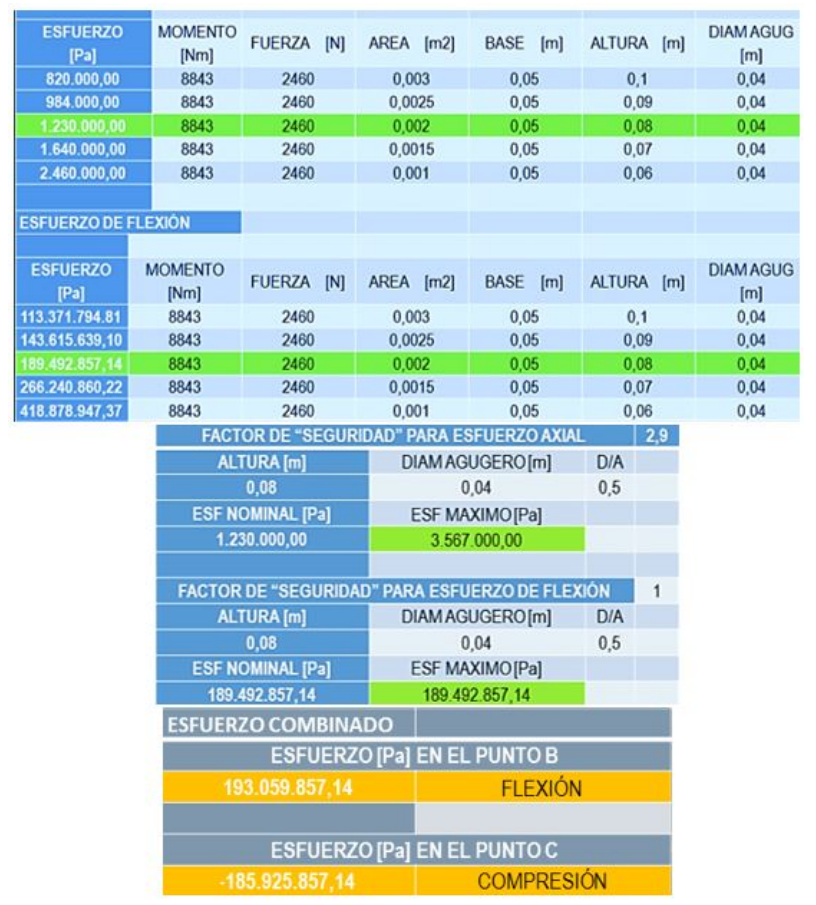

Para garantizar el funcionamiento de los elementos se realiza simulaciones en software INVENTOR (Figura 10) y se verifica los esfuerzos resultantes.

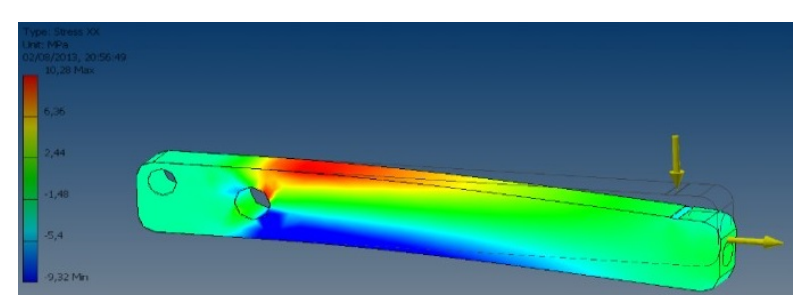

Figura 10. Simulación de esfuerzos en brazo de doblado

Al disponer de este software y de piezas de formas especiales como los triángulos soportes (Figura 11) se 
Pérez-Silva, Trujillo-Zurita. / Diseño de una máquina para cortar y doblar estribos en serie para columnas de

puede simular la carga y obtener el esfuerzo necesario para determinar el material de construcción.

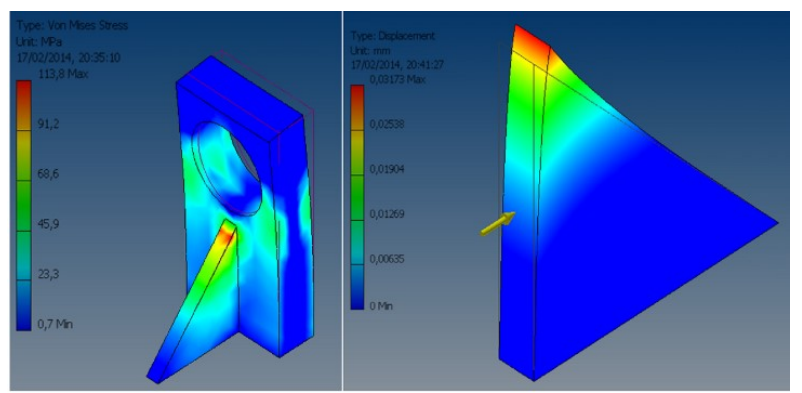

Figura 11. Simulación de esfuerzos en triángulos soporte

Como un plus al cálculo de elementos especiales como el mencionado, se realiza una combinación de cálculos tomando como referencia una viga empotrada en su extremo (Figura 12), con sección rectangular, pero analizada por puntos críticos.
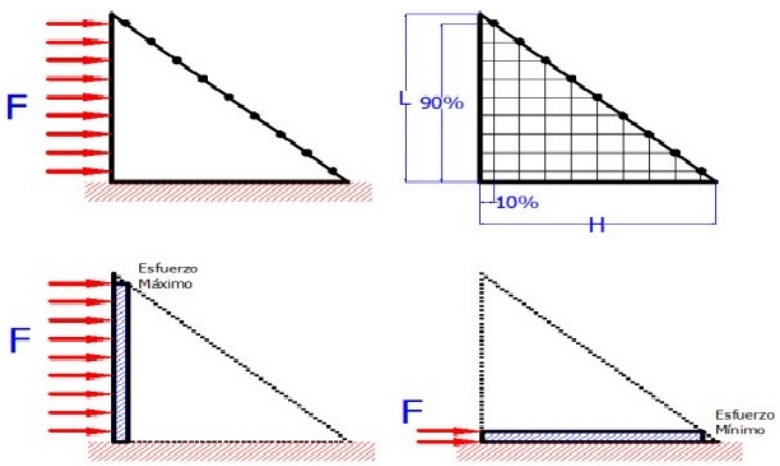

Figura 12. Análisis matemático por puntos críticos

La simulación se comprueba mediante una tabla (Tabla 4) de Excel que hace referencia a los porcentajes en distancia de los dos esquemas de viga empotrada.

Tabla 4. Tabla de cálculo porcentual combinado para una simulación de triángulos

\begin{tabular}{|c|c|c|c|c|}
\hline \multicolumn{5}{|c|}{ CÁLCULO DE ESFUERZOS EN UNA PIEZA TRIANGULAR } \\
\hline CARGA [N] & \begin{tabular}{|l|} 
DIST "L" \\
{$[\mathrm{m}]$}
\end{tabular} & \begin{tabular}{|l|} 
DIST H \\
{$[\mathrm{m}]$} \\
\end{tabular} & & \\
\hline 2830 & 0,035 & 0,035 & & \\
\hline DISTRIBUCIÓN [\%] & \begin{tabular}{|l|} 
DIST "L" \\
[m]
\end{tabular} & \begin{tabular}{|l} 
DIST H \\
{$[\mathrm{m}]$}
\end{tabular} & \begin{tabular}{|l} 
ESPESOR \\
{$[\mathrm{m}]$}
\end{tabular} & ESFUERZO [Pa] \\
\hline 90 & 0,0315 & 0,0035 & 0,005 & $275.076 .000,0$ \\
\hline 80 & 0,028 & 0,007 & 0,005 & $54.336 .000,0$ \\
\hline 70 & 0,0245 & 0,0105 & 0,005 & $18.489 .333,3$ \\
\hline 60 & 0,021 & 0,014 & 0,005 & $7.641 .000,0$ \\
\hline 50 & 0,0175 & 0,0175 & 0,005 & $3.396 .000,0$ \\
\hline 40 & 0,014 & 0,021 & 0,005 & $1.509 .333,3$ \\
\hline 30 & 0,0105 & 0,0245 & 0,005 & $623.755,1$ \\
\hline 20 & 0,007 & 0,028 & 0,005 & $212.250,0$ \\
\hline 10 & 0,0035 & 0,0315 & 0,005 & $41.925,9$ \\
\hline
\end{tabular}

Las partes restantes y piezas normalizadas se determinaron bajo parámetros estandarizados que facilitan el cálculo y la fiabilidad de los elementos en estudio. Todos los elementos y partes se diseñaron con un factor de seguridad de dos (2) por cuanto se trabaja con cargas dinámicas [6], para evitar los altos incrementos de costo por sobremedidas y mecanizados, garantizando la funcionalidad y durabilidad. Así, por ejemplo, se determina para el cálculo de pernos (Figura 13) todas las variables necesarias, desde las características del elemento hasta la carga final que puede soportar en tracción y el factor de seguridad que garantiza la junta.

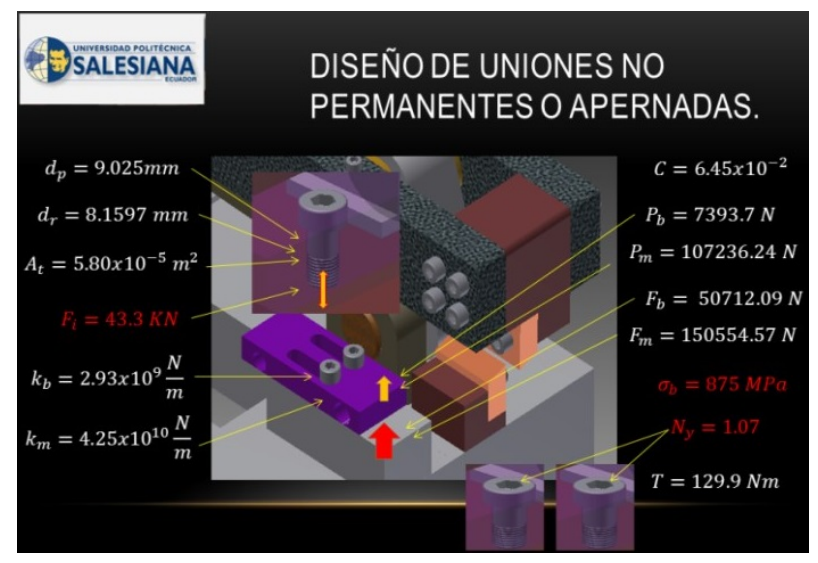

Figura 13. Referencias necesarias para el cálculo de per$\operatorname{nos}[7]$

\subsection{Funcionamiento y partes}

La máquina que se ha diseñado y construido para cortar y doblar estribos consta de un motor con potencia de $2,2 \mathrm{~kW}$ que entrega un torque de $(670 \mathrm{Nm})$ mediante un reductor de velocidad cuya relación de transmisión es de $1 / 56$. Este torque, que genera un movimiento rotativo es convertido a un movimiento lineal gracias a un mecanismo denominado cigüeñal-biela (Figura 14).

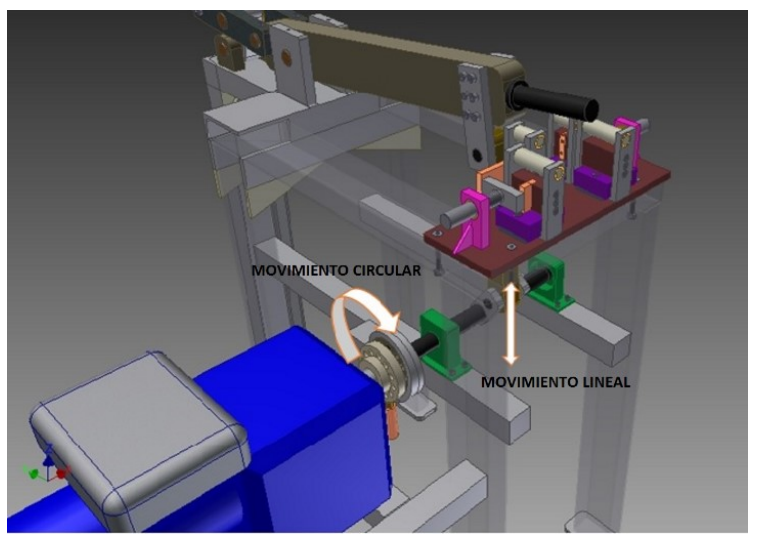

Figura 14. Mecanismo cigüeñal-biela que transforma el movimiento circular en lineal 
La fuerza es transmitida hacia el brazo de doblado y este a su vez genera movimiento en los eslabones posteriores, los cuales se mueven y crean reacciones necesarias para cortar la varilla corrugada en el extremo posterior de la máquina (Figura 15).

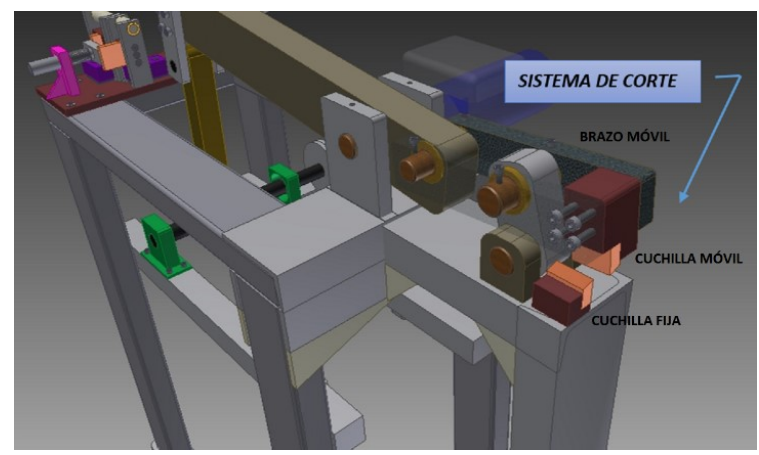

Figura 15. Sistema de corte

Las principales partes de la máquina son los sistemas de corte y doblado (Figura 16), los cuales son regulables a las distancias necesarias, pero limitadas. El proyecto concluye realizando las pruebas extremas para cortar y doblar varilla de diámetro $8 \mathrm{~mm}$ obteniendo productos con mejores acabados que los hechos a mano.

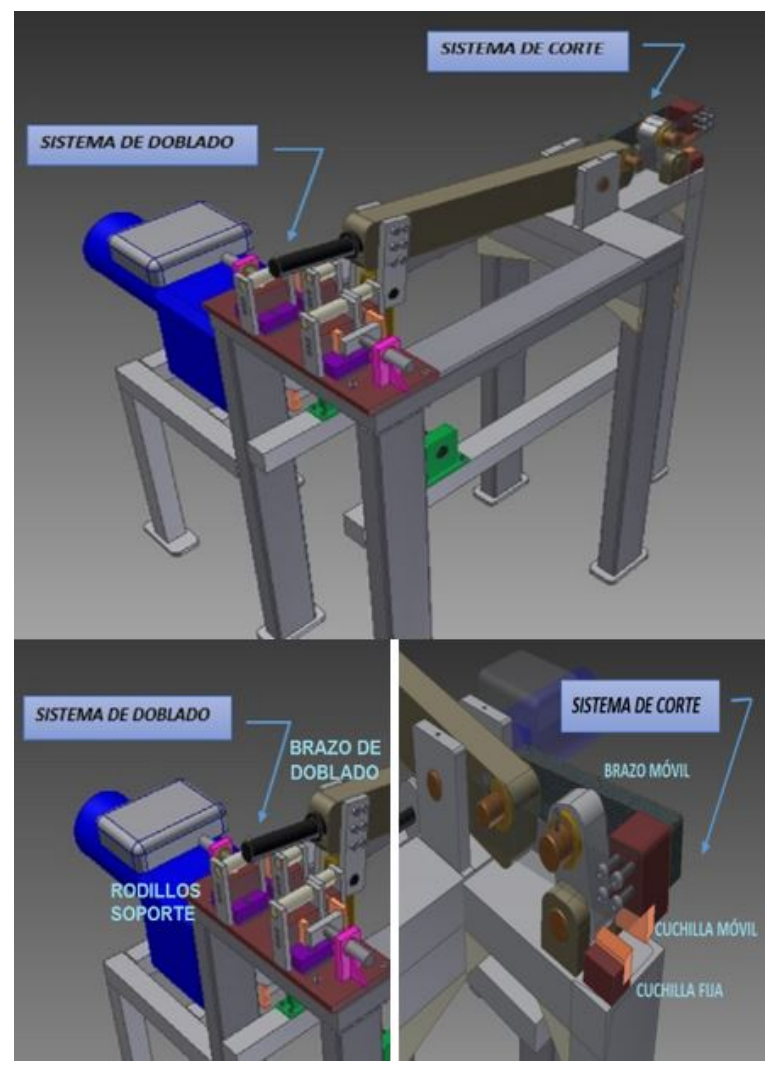

Figura 16. Mecanismo de doblado y corte

\section{Resultados y discusión}

La máquina cumple el objetivo principal que está planteado, cortar y doblar varilla corrugada para realizar estribos en serie de diámetro $8 \mathrm{~mm}$.

En pruebas de funcionamiento, se obtuvieron 600 estribos en aproximadamente dos horas de trabajo lo que incrementa la producción en un $400 \%$, además, con una mejora notable en acabados y formas, que superaron ampliamente a los obtenidos manualmente (Figura 17), también implica que el obrero puede aprovechar alrededor de 6 horas en otra actividad, evitando así la fatiga laboral y disminuyendo costos de operación.

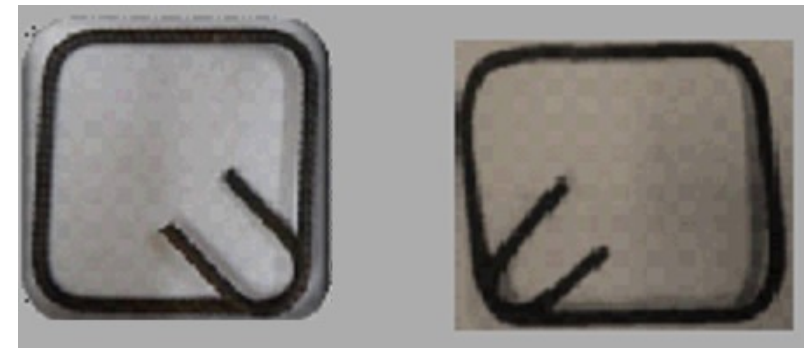

Figura 17. Estribo realizado con máquina y estribo realizado a mano respectivamente

Mediante el análisis RULA se verifica que los índices de esfuerzos bajan de 7 a 3 en los operarios que realizaban el trabajo manual, evitando así una enfermedad ocupacional.

\section{Conclusiones}

Emprender un proyecto es buscar la solución a un problema que inicia con la necesidad de ingeniar algo nuevo o cambiar algo que ya está definido, el segundo paso es analizar todo lo ya existente dándole un giro de 360 grados. Cuando se da sentido a una idea se la define y limita, de manera que las metas sean cuantitativas y cualitativas y todo proyecto se encamine en una misma línea de acción.

Realizar maquetas y pruebas con elementos reales y a escala es parte de la investigación experimental y se constituye en gran ayuda para despejar dudas que los cálculos y simulaciones nos suelen proponer.

El uso de tablas de cálculo iterativas y nuevos softwares facilitan la definición de una forma constructiva así como garantizan el material que se debe utilizar bajo ciertas cargas de trabajo.

Dar solución a problemas prácticos de la vida cotidiana contribuye al desarrollo ingenieril y tecnológico del país, ya que disminuye tiempo que se traduce en dinero y apoya al desarrollo de las actividades productivas. 


\section{Referencias}

[1] INEC. Instituto ecuatoriano de estadísticas y censos. [Online]. Available: www.inec.gob.ec

[2] A. Arequipa. (2014) Manual para propietarios. Aceros Arequipa S. A. [Online]. Available: http://www.acerosarequipa.com/ manual-para-propietarios.html

[3] C. D. I. D. Ejército. [Online]. Available: www.cuerpodeingenierosdelejercito.mil.ec
[4] O. Ergonomics. Osmond Group Limited. [Online]. Available: http://www.rula.co.uk

[5] R. Timoshenko, Mecánica de Materiales. México: Pearson Education, 2011.

[6] R. Mott, Diseño de elementos de máquinas. México: Pearson Education, 2006.

7] R. Norton, Diseño de máquinas. México: Pearson Education, 2011. 